\title{
Discrepant NOXA (PMAIP1) transcript and NOXA protein levels: a potential Achilles' heel in mantle cell lymphoma
}

\author{
MA Dengler ${ }^{1}$, A Weilbacher ${ }^{1}$, M Gutekunst ${ }^{1}$, AM Staiger ${ }^{1}$, MC Vöhringer ${ }^{2}$, H Horn ${ }^{1}$, G Ott ${ }^{3}$, WE Aulitzky ${ }^{2}$ and H van der Kuip*,1
}

Mantle cell lymphoma (MCL) is an aggressive lymphoid neoplasm with transient response to conventional chemotherapy. We here investigated the role of the Bcl-2 homology domain 3-only protein NOXA for life-death decision in MCL. Surprisingly, NOXA (PMAIP1) mRNA and NOXA protein levels were extremely discrepant in MCL cells: NOXA mRNA was found to be highly expressed whereas NOXA protein levels were low. Chronic active B-cell receptor signaling and to a minor degree cyclin D1 overexpression contributed to high NOXA mRNA expression levels in MCL cells. The phoshatidyl-inositol-3 kinase/AKT/ mammalian target of rapamycin pathway was identified as the major downstream signaling pathway involved in the maintenance of NOXA gene expression. Interestingly, MCL cells adapt to this constitutive pro-apoptotic signal by extensive ubiquitination and rapid proteasomal degradation of NOXA protein $\left(T_{1 / 2} \sim 15-30 \mathrm{~min}\right)$. In addition to the proteasome inhibitor Bortezomib, we identified the neddylation inhibitor MLN4924 and the fatty acid synthase inhibitor Orlistat as potent inducers of NOXA protein expression leading to apoptosis in MCL. All inhibitors targeted NOXA protein turnover. In contrast to Bortezomib, MLN4924 and Orlistat interfered with the ubiquitination process of NOXA protein thereby offering new strategies to kill Bortezomib-resistant MCL cells. Our data, therefore, highlight a critical role of NOXA in the balance between life and death in MCL. The discrepancy between NOXA transcript and protein levels is essential for sensitivity of MCL to ubiquitin-proteasome system inhibitors and could therefore provide a druggable Achilles' heel of MCL cells.

Cell Death and Disease (2014) 5, e1013; doi:10.1038/cddis.2013.552; published online 23 January 2014

Subject Category: Cancer

Mantle cell lymphoma (MCL) is a lymphoid neoplasm characterized by abnormal proliferation of mature B-lymphocytes derived from the mantle zone of lymphoid follicles. ${ }^{1,2} \mathrm{MCL}$ accounts for $5-10 \%$ of all non-Hodgkin lymphomas and usually shows an aggressive clinical course with comparatively short response to chemotherapy, frequent relapses and a median survival of $5-7$ years. ${ }^{1,3}$ The genetic hallmark of $M C L$ is the $t(11 ; 14)(q 13 ; q 32)$ translocation leading to cyclin D1 overexpression. This alteration causes cell cycle deregulation and is considered the primary oncogenic event in MCL pathogenesis. ${ }^{1,2} \mathrm{MCL}$ cells also carry a high number of secondary genetic alterations further disturbing cell cycle regulation and/or DNA damage response. ${ }^{3,4}$ In addition, deregulation of different oncogenic signaling pathways involved in proliferation and/or survival, ${ }^{2,3}$ such as constitutive activation of B-cell receptor (BCR), ${ }^{5}$ phoshatidyl-inositol-3 kinase (PI3K)/ AKT/mammalian target of rapamycin (mTOR) ${ }^{6}$ and NF- $\mathrm{KB}$ signaling pathways, ${ }^{7}$ and defects in apoptosis regulation ${ }^{8,9}$ have been described in MCL.
Targeting these pathways may provide new therapeutic strategies in MCL. First studies using the BTK inhibitor Ibrutinib or the $\mathrm{PI} 3 \mathrm{~K} / \mathrm{mTOR}$ inhibitor Bez235 showed promising effects. ${ }^{10,11}$ Inhibitors of the ubiquitin-proteasome system (UPS) such as Bortezomib (Velcade) also recently entered the clinic and showed efficacy in MCL. ${ }^{12}$ The mechanism by which Bortezomib induces cell death appears to differ between tumor entities. In $\mathrm{MCL}$, the $\mathrm{Bcl}-2$ homology domain 3 (BH3)-only protein NOXA seems to be an important mediator of apoptosis upon Bortezomib. ${ }^{13}$ NOXA is a 'sensitizer' BH3-only protein, which mainly acts by targeting the anti-apoptotic protein Mcl1. ${ }^{14,15}$ It is highly regulated at transcriptional ${ }^{16}$ and post-transcriptional levels. Recent studies have shown that NOXA protein is regulated by ubiquitination and proteasomal degradation. ${ }^{17,18}$

NOXA is thought to be crucial in order to fine-tune apoptotic signaling. ${ }^{16}$ Enhanced NOXA protein turnover, for example, can result in resistance to genotoxic stress. ${ }^{18}$ We recently demonstrated that high constitutive NOXA levels are central for hypersensitivity of embryonal carcinoma cells to cisplatin. ${ }^{19}$

\footnotetext{
${ }^{1}$ Dr. Margarete Fischer-Bosch Institute of Clinical Pharmacology, Stuttgart, and University of Tübingen, Auerbachstr. 112, Stuttgart 70376, Germany; ${ }^{2}$ Second Department of Internal Medicine, Oncology and Hematology, Robert-Bosch-Hospital, Auerbachstr. 110, Stuttgart 70376, Germany and ${ }^{3}$ Department of Clinical Pathology, Robert-Bosch-Hospital, Auerbachstr. 110, Stuttgart 70376, Germany

${ }^{*}$ Corresponding author: H van der Kuip, Dr. Margarete-Fischer Bosch Institute of Clinical Pharmacology, Auerbachstr. 112, Stuttgart 70376, Germany. Tel: +49 7118101 3730; Fax: +49 711 859295; E-mail: heiko.van-der-kuip@ikp-stuttgart.de

Keywords: NOXA (PMAIP1); mantle cell lymphoma; ubiquitin-proteasome system; apoptosis

Abbreviations: ALCL, anaplastic large cell lymphoma; BCR, B-cell receptor; BH3, Bcl-2 homology domain 3; BTK, Bruton's tyrosine kinase; CD, cluster of differentiation; CLL, chronic lymphoid leukemia; CRL, cullin-RING ubiquitin ligases; FASN, fatty acid synthase; MCL, mantle cell lymphoma; mTOR, mammalian target of rapamycin; PI3K, phoshatidyl-inositol-3 kinase; siRNA, small interfering ribonucleic acid; TUBE, tandem ubiquitin binding entities; UPS, ubiquitin-proteasome system Received 14.9.13; revised 12.12.13; accepted 13.12.13; Edited by M Agostini
} 
Besides its role in cellular stress response, NOXA is a critical mediator of B-cell development mediating apoptosis in activated $B$ cells. ${ }^{20,21}$ Considering this and its important role for the response of MCL cells to Bortezomib, NOXA may be a central determinant in the regulation of life-death decision in MCL and represent a key target for treatment. A better understanding of the mechanism how NOXA is regulated may offer new and more effective strategies for therapy of this aggressive malignancy.

\section{Results}

MCL cells are characterized by high constitutive NOXA gene expression but low levels of NOXA protein. To compare NOXA transcript levels in MCL cells with other cancer entities, we used the MCL cell lines Mino and Rec1 characterized by different p53 status (Mino: mutant; Rec1; wild type) and a collection of cancer cell lines derived from embryonal carcinoma, lung and ovarian cancer. Interestingly, NOXA transcript was significantly higher in MCL cell lines when compared with the other cell lines and PBMCs of healthy donors (Figure 1a). We then compared NOXA transcript levels of the MCL cell lines Mino, Rec1, Jvm2, Granta519 and Jeko1 with samples from MCL patients. All four primary MCL samples displayed similar or even higher constitutive NOXA mRNA levels as compared with MCL cell lines (Figure 1b).
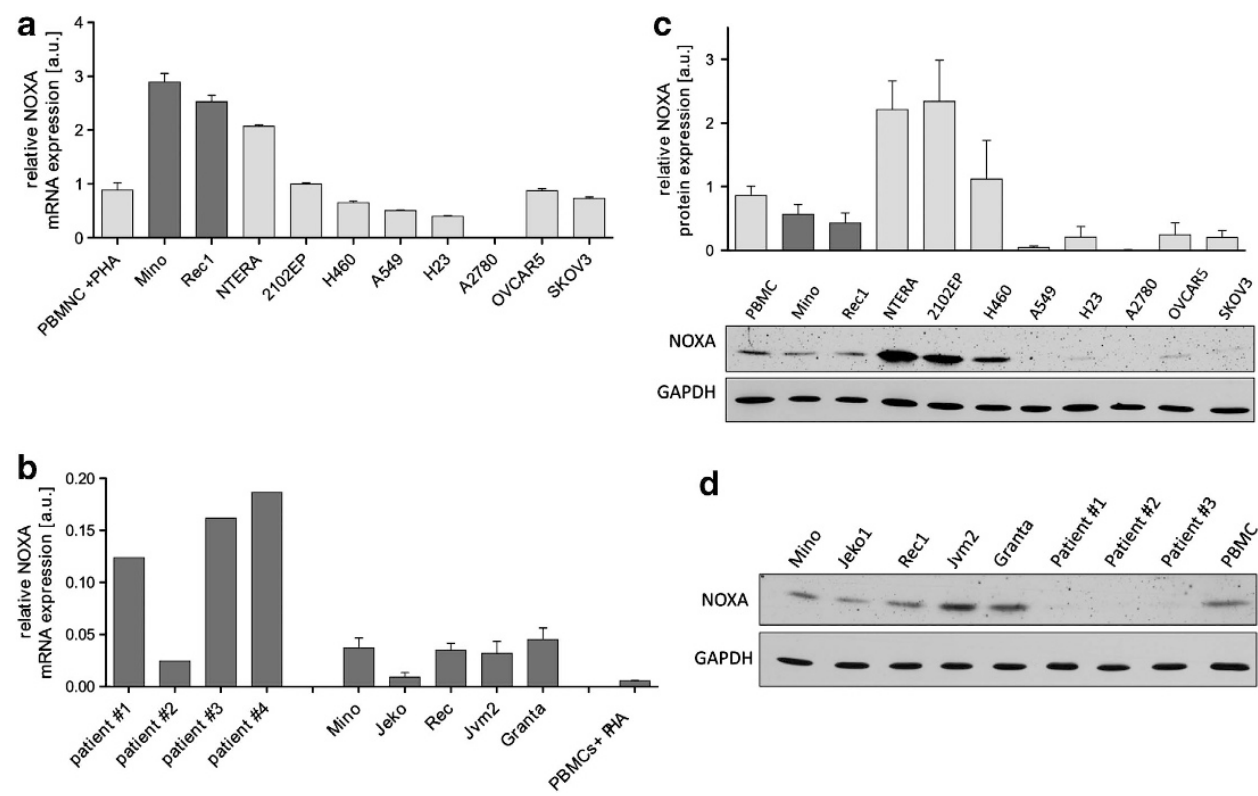

d

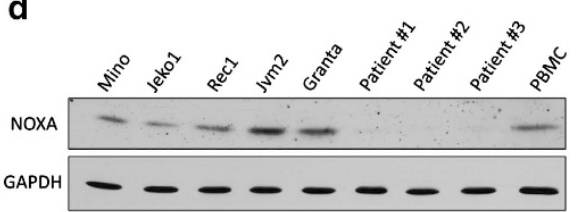

To assess the relevance of the observed differences in NOXA mRNA levels in this small cell line panel, we used data from the Cancer Cell Line Encyclopedia (CCLE), ${ }^{22}$ a database providing whole-genome gene expression profiles of a large collection of cancer cell lines.

Interestingly, NOXA mRNA expression in cell lines derived from hematologic malignancies was the second highest of all tumor entities (Supplementary Figure 1a). Among blood cancers, MCL cells together with anaplastic large cell lymphoma (ALCL) and chronic lymphoid leukemia (CLL) expressed the highest level of NOXA transcripts (Supplementary Figure 1b) and the MCL cell line Granta519 even showed the highest NOXA expression in the panel of $>1000$ cell lines available from CCLE (Supplementary Figure 1c). This observation indicates that NOXA indeed has an important role in the regulation of life-death decision in $\mathrm{MCL}$.

As NOXA expression is regulated both at transcriptional ${ }^{16}$ and post-transcriptional levels, ${ }^{17,18}$ we next analyzed NOXA protein levels in the same panel of cell lines previously used for gene expression analysis in Figure 1a. Importantly, the pattern of NOXA protein expression was not correlated to mRNA expression (Figure 1c). The level of NOXA protein, in contrast to the high transcript levels, was found to be relatively low in MCL cell lines. In samples derived from MCL patients, NOXA protein was hardly detectable and the discrepancy between RNA and protein levels was even more pronounced (Figure 1d).

Figure $1 \mathrm{MCL}$ cells express high levels of NOXA (PMAIP1) mRNA but low NOXA protein. (a) Analysis of NOXA gene expression in MCL cell lines Mino and Rec1 compared with embryonal carcinoma cell lines (NTERA2/D1, 2102EP) lung cancer cell lines (A549, NCI-H23, NCI-H460), ovarian cancer cell lines (OVCAR5, SKOV3, A2780) and PHA-stimulated PBMCs of healthy donors using high-throughput real-time PCR BioMark HD System. NOXA mRNA expression was normalized to TBP. Data represent means \pm S.D. from three technical replicates. (b) Comparison of NOXA mRNA levels in samples from four MCL patients with the MCL cell lines Mino, Jeko1, Rec1, Jvm2 and Granta519 and PBMCs of healthy donors by conventional real-time PCR. NOXA mRNA expression was normalized to GAPDH. Data of MCL cell lines and PBMCs represent means \pm S.D. from three experiments. (c) MCL cell lines have relatively low constitutive NOXA protein expression. Comparison of NOXA protein expression levels in MCL cell lines Mino and Rec1 compared with embryonal carcinoma cell lines (NTERA2/D1, 2102EP) lung cancer cell lines (A549, NCl-H23, NCl-H460), ovarian cancer cell lines (OVCAR5, SKOV3, A2780) and PHA-stimulated PBMCs of healthy donors. Upper panel: densitometric evaluation of NOXA protein expression analyzed by western blot. NOXA protein expression was normalized to GAPDH. Data reflect means \pm S.D. from three experiments. Lower panel: representative western blot of NOXA protein expression. (d) Constitutive NOXA protein expression in MCL cell lines Mino, Jeko1, Rec1, Jvm2 and Granta519 compared with primary MCL samples and PHA-stimulated PBMCs of healthy donors analyzed by western blot 
High NOXA transcript levels depend on constitutive PI3K/AKT/mTOR signaling. NOXA mRNA is upregulated in activated normal B cells upon BCR ligation. ${ }^{20}$ Constitutive activation of the BCR is also a feature of MCL. ${ }^{5}$ To elucidate the impact of BCR-mediated signaling on NOXA transcript levels in MCL cells, we performed RNAi-mediated knockdown of CD79A (cluster of differentiation 79A), an essential part of the BCR. Disruption of BCR activation reduced phosphorylation of downstream effector kinases ERK, AKT, $\mathrm{I} \mathrm{KB}$ and S6 (Figure 2a, upper panel) and significantly decreased NOXA transcript levels (Figure 2a, lower panel). These results were supported by similar effects upon pharmacological inhibition of the BCR pathway using Ibrutinib an inhibitor of the BCR-associated kinase BTK (Supplementary Figure 2a). To test which of the BCR downstream pathways mediates the high NOXA transcript levels in $\mathrm{MCL}$, we targeted each pathway using selective inhibitors or RNAi. Interestingly, inhibition of the MAPK pathway by PD0325901 as well as knockdown of p65 (RELA), the main downstream effector of $N F-\kappa B$ signaling, had only minimal effects on the level of NOXA mRNA or even enhanced NOXA transcription (Figure $2 \mathrm{~b}$, upper and middle panel). In contrast, inhibition of the PI3K/AKT/mTOR pathway by the dual PI3K/mTOR inhibitor Bez235 strongly reduced NOXA transcripts (Figure $2 \mathrm{~b}$, lower panel). Further experiments using different inhibitors of the PI3/AKT/mTOR pathway supported this finding, as all of them reduced NOXA transcript with highest efficacy when PI3K and mTOR inhibitors were combined, indicating that both $\mathrm{PI} 3 \mathrm{~K}$ and mTOR are essential for maintaining high NOXA transcription (Supplementary Figure 2b).

We next investigated whether the aberrant expression of cyclin D1 influences NOXA gene expression. RNAi-mediated downregulation of cyclin D1 showed similar results as observed for knockdown of CD79A although not that pronounced. Remarkably, reduced cyclin D1 expression led to a partial inhibition/reduced expression of AKT and/orS6 (Figure 2c, upper panel), which may be responsible for the decrease in NOXA mRNA levels (Figure 2c, lower panel). This hypothesis was supported by the finding that cyclin D1 knockdown did not further reduce NOXA mRNA in cells treated with Bez235 indicating that both target the same pathway (Supplementary Figure 2c).

To elucidate whether constitutive activation of the PI3K/ AKT/mTOR pathway is also present in primary MCL cells, we compared PI3K and mTOR activity in MCL cell lines, primary MCL samples and stimulated PBMCs of healthy donors. Cell lines as well as primary MCL cells showed significant phosphorylation of AKT and/or S6 (Figure 2d, upper panel). Inhibition of the PI3K/AKT/mTOR pathway by Bez235 led to a strong reduction of NOXA mRNA expression in primary MCL cells (Figure 2d, lower panel) further supporting the previous findings in the MCL cell lines.

Low NOXA protein levels are caused by a short half-life of the NOXA protein in MCL cells. To determine if the discrepancy between NOXA transcript and protein levels is due to increased NOXA turnover, we analyzed NOXA protein stability. Compared with PBMCs of healthy donors and a panel of cancer cell lines NOXA protein stability was found to be significantly lower in MCL cells (Figure 3a, Supplementary Figure 3) with a half-life of 15-30 min (Figure 3a). Remarkably, NOXA protein turnover was not affected by inhibition of MAPK, PI3K/AKT/mTOR and NF- $\mathrm{KB}$ signaling indicating that NOXA protein stability is not regulated by either of these pathways (Supplementary Figure 4a). Owing to the reduction of NOXA mRNA, the low NOXA protein levels were even further reduced by inhibition of BCR signaling as well as knockdown of cyclin D1 (Supplementary Figure 4b).

It was recently shown, that NOXA is a target of the UPS. ${ }^{18}$ We therefore investigated if the short half-life of NOXA in MCL is mediated by polyubiquitination and proteasomal degradation. As shown in Figure 3b, tandem ubiquitin binding entity 2 (TUBE2) pull-down of polyubiquitinated proteins and western blot analysis revealed that NOXA is extensively ubiquitinated and degraded by the proteasome as inhibition of the proteasome by MG132 or Lactacystin led to accumulation of NOXA and its polyubiquitinated forms. These results show that although NOXA is transcriptionally highly expressed in MCL cells, NOXA protein expression is low because of its rapid turnover.

Agents that cause accumulation of NOXA protein potently kill MCL cells in a NOXA-dependent manner. To identify substances able to induce or stabilize NOXA in MCL cells, we screened several known and potential NOXA inducers and agents, which have been shown to be effective in MCL for their ability to accumulate NOXA. In accordance with recently published data, ${ }^{13,23}$ the proteasome inhibitor Bortezomib very effectively accumulated NOXA protein. A similar effect was also observed upon treatment with MLN4924, an inhibitor of neddylation, which is required for activity of several E3 ubiquitin ligases (Figure 4a). Surprisingly, the fatty acid synthase (FASN) inhibitor Orlistat, which has been shown to kill MCL cell lines, ${ }^{24}$ also led to significantly elevated NOXA protein levels. Of note, Bortezomib, MLN4924 and Orlistat also accumulated NOXA protein in primary $\mathrm{MCL}$ cells (Supplementary Figure 5). In contrast, known transcriptional NOXA inducers such as the DNA-damaging agents Cisplatin ${ }^{25}$ or Doxorubicin ${ }^{26}$ were not able to induce NOXA protein in both mt-p53 (Mino) and wt-p53 (Rec1) MCL lines (Figure 4a). Cell death was most efficiently induced by compounds causing NOXA protein accumulation (Figure 4b). Although NOXA accumulation was less pronounced upon MLN4924, the efficacy in induction of cell death was as efficient as observed upon Bortezomib or Orlistat. This is likely due to the lack of Mcl1 induction, the anti-apoptotic antagonist of NOXA, which was observed upon treatment with Bortezomib and Orlistat (Supplementary Figure 6).

Interestingly, dose-response curves showed that Orlistat and MLN4924 may be more selective than Bortezomib in MCL cells. PBMCs and fibroblasts from healthy donors responded only at highest doses of Orlistat and did not respond at all to MLN4924 (Figure 4c). Moreover, in contrast to Bortezomib and MLN4924, knockdown of cyclin D1 substantially rescued cells from Orlistat-induced apoptosis (Supplementary Figure 7a) and reduced NOXA accumulation (Supplementary Figure 7b) indicating that Orlistat may be more selective for cyclin D1-overexpressing MCL cells. To confirm the role of NOXA for 
a
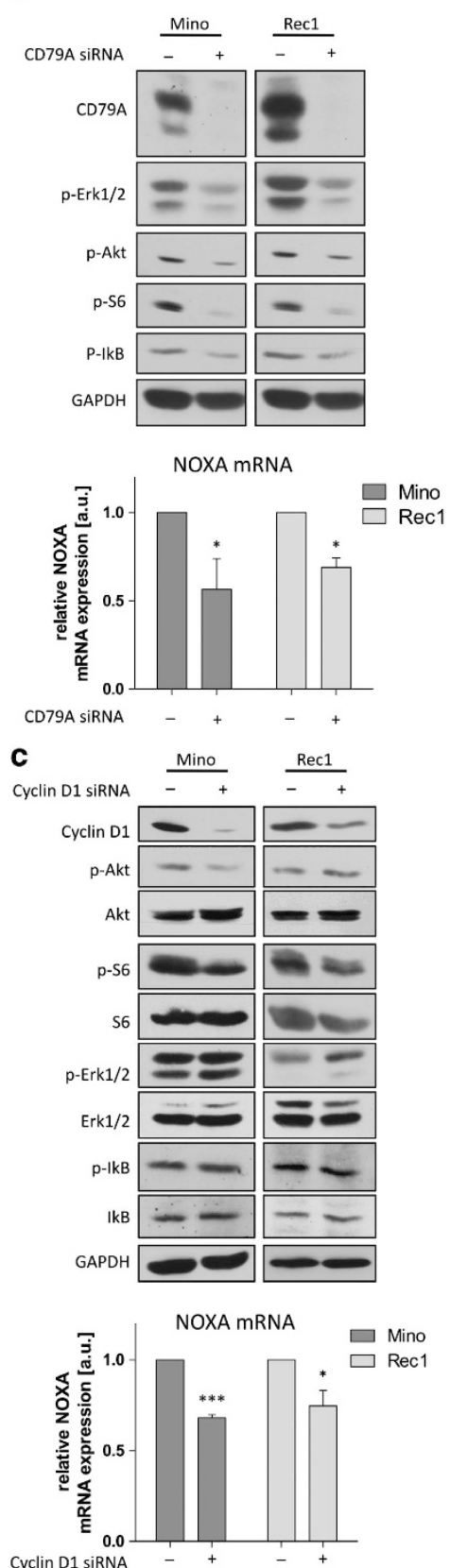

b
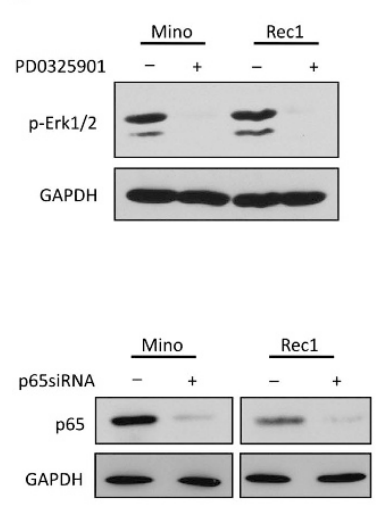

GAPDH

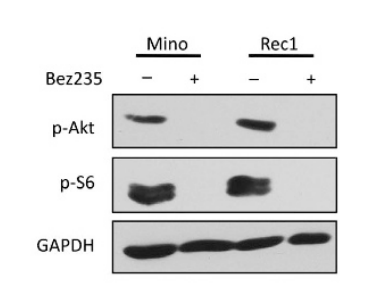

d

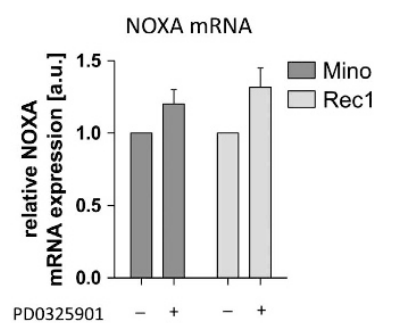

PD0325901 -7 Mino
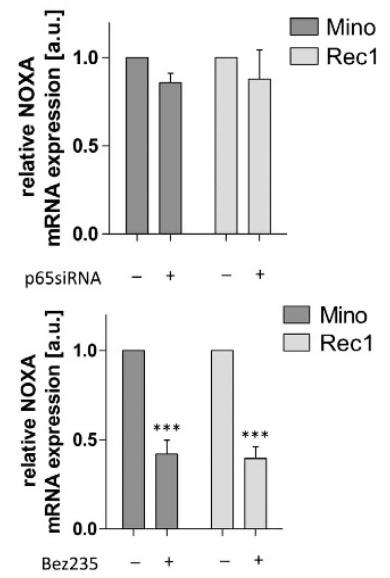

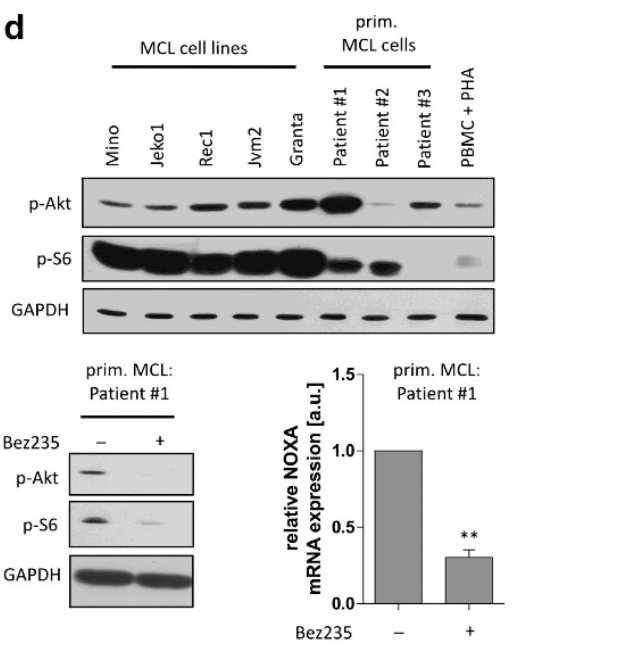

Figure $2 \mathrm{BCR}$ and cyclin D1 mediate the high constitutive NOXA mRNA levels via the PI3/AKT/mTOR pathway. (a) Inhibition of BCR signaling causes reduction of constitutive NOXA mRNA expression in MCL cell lines Mino and Rec1. Effect of RNAi-mediated silencing of CD79A on BCR downstream pathways (upper panel) and constitutive expression of NOXA mRNA (lower panel; ${ }^{*} P<0.05$ ). Cells were transfected with control siRNA or siRNA targeting CD79A. After $24 \mathrm{~h}$, cells were harvested for western blot analysis and quantification of NOXA mRNA levels. NOXA mRNA expression was normalized to GAPDH. Data represent means \pm S.D. from three experiments. (b) Impact of inhibition of BCR downstream pathways on constitutive NOXA expression. Upper panel: inhibition of the MAPK pathway has only minor effects on NOXA mRNA levels. Cell lines Mino and Rec1 were treated with $1 \mu \mathrm{M}$ of PD0325901 for $6 \mathrm{~h}$ then harvested for western blot analysis and quantification of NOXA mRNA. Middle panel: effect of p65 knockdown on constitutive NOXA expression in MCL cell lines Mino and Rec1. Cells were transfected with control siRNA or siRNA targeting $p 65$. After $48 \mathrm{~h}$, cells were harvested for western blot analysis and quantification of NOXA mRNA levels. Lower panel: inhibition of the PI3K/AKT/mTOR pathway significantly reduces NOXA mRNA and protein expression. Cell lines Mino and Rec1 were treated with $1 \mu \mathrm{M}$ of Bez235 for $6 \mathrm{~h}$ then harvested for western blot analysis and quantification of NOXA mRNA levels $\left.{ }^{* \star \star} P<0.001\right)$. NOXA mRNA expression was normalized to GAPDH. All data represent means \pm S.D. from three experiments. (c) Knockdown of cyclin D1 attenuates PI3K/AKT/mTOR signaling and reduces constitutive NOXA mRNA levels in MCL cell lines Mino and Rec1. Cells were transfected with control siRNA or siRNA targeting cyclin D1 (CCND1). After $16 \mathrm{~h}$, cells were harvested for western blot (upper panel) analysis and quantification of NOXA mRNA levels (lower panel; ${ }^{*} P<0.05,{ }^{* * *} P<0.001$ ). NOXA mRNA expression was normalized to GAPDH. Data represent means \pm S.D. from three experiments. (d) Upper panel: comparison of PI3K/AKT/mTOR signaling in MCL cell lines, primary MCL samples and PBMCs of healthy donors. Phosphorylation of PI3K downstream kinases was measured by western blot. Lower panel: effect of PI3K/AKT/mTOR pathway inhibition on constitutive NOXA mRNA expression in MCL patients. Primary MCL cells were treated with $1 \mu \mathrm{M}$ of Bez235 for $6 \mathrm{~h}$ then harvested for western blot analysis and quantification of NOXA mRNA levels ( ${ }^{* \star} P<0.01$ ). NOXA mRNA expression was normalized to GAPDH. Data represent means \pm S.D. from three technical replicates 

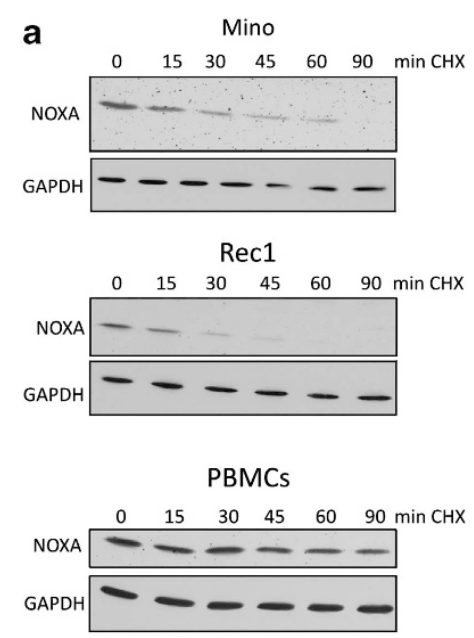

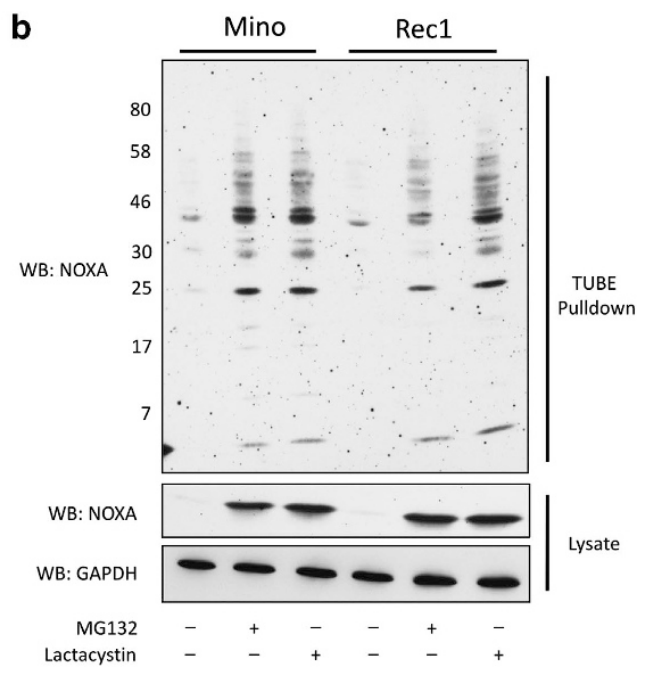

Figure 3 Rapid UPS-mediated NOXA protein turnover in MCL cells. (a) Half-life of NOXA protein in MCL cell lines (Mino, Rec1) and PHA-stimulated PBMCs of healthy donors. Cells were exposed to $20 \mu \mathrm{g} / \mathrm{ml}$ cycloheximide and half-life of NOXA was determined by western blot. (b) NOXA is polyubiquitinated and degraded by the proteasome. Cells were cultivated in absence or presence of $1 \mu \mathrm{M} \mathrm{MG132}$ or $5 \mu \mathrm{M}$ lactacystin for $8 \mathrm{~h}$ then harvest and lysed. Total polyubiquitinated proteins were isolated from cell lysates using agarose-TUBE2 beads. Protein expression and polyubiquitination state of NOXA were analyzed by western blot analysis using an NOXA-specific antibody

induction of cell death upon Bortezomib, Orlistat and MLN4924 treatment, we silenced NOXA via RNAi. Indeed, NOXA knockdown significantly rescued the cells from apoptosis and reduced NOXA protein accumulation upon treatment (Figure 4d).

As the PI3K/AKT/mTOR pathway is involved in regulation of constitutive NOXA levels in MCL cells and its inhibition significantly reduces NOXA mRNA (Figure 2), this pathway may also have an impact on accumulation of NOXA protein and induction of cell death upon treatment with Bortezomib, Orlistat and MLN4924. To test this hypothesis, we preincubated the MCL cells with Bez235 before treatment with the respective inhibitors. Interestingly, we observed an effect comparable to that upon RNAi-mediated knockdown of NOXA. Inhibition of PI3K/AKT/mTOR pathway reduced UPS inhibitor mediated induction of apoptosis and accumulation of NOXA (Figure 4e). A similar effect could be observed by pretreatment of the cells with Ibrutinib, which inhibits the main upstream pathway of PI3K/AKT/mTOR (Supplementary Figure 8). These findings indicate that a constitutively active $\mathrm{PI} 3 \mathrm{~K} / \mathrm{AKT} / \mathrm{mTOR}$ pathway is needed to effectively kill MCL cells through accumulation of NOXA.

Orlistat and MLN4924 stabilize NOXA by inhibition of NOXA ubiquitination and efficiently kill Bortezomibresistant MCL cells. Our previous results indicate that Bortezomib, Orlistat and MLN4924 rather target the high NOXA protein turnover than transcriptionally activate NOXA. Indeed, Bortezomib, Orlistat as well as MLN4924 significantly enhanced NOXA protein stability (Figure 5a; Supplementary Figure 9a). We next investigated their ability to accumulate polyubiquitinated NOXA species by TUBE2 pull-down experiments. Importantly, in contrast to Bortezomib neither Orlistat nor MLN4924 led to accumulation of slower migrating polyubiquitinated forms of NOXA (Figure 5b; Supplementary Figure 9b). These results suggest that Orlistat and MLN4924 inhibit NOXA ubiquitination rather than the proteasome. Bortezomib-resistant cells might therefore still be sensitive to these alternative NOXA-stabilizing agents. To test this hypothesis, we treated Bortezomibsensitive MCL cells (Jeko1-BS) and Bortezomib-resistant cells (Jeko1-BR) with Orlistat or MLN4924. As expected, Jeko1-BS cells were sensitive to Orlistat and MLN4924 and induced NOXA protein (Figure 5c, left panel). Remarkably, the Bortezomib-resistant clone Jeko1-BR, which showed no signs of cell death when treated with Bortezomib, was still sensitive to Orlistat and MLN4924. Bortezomib-mediated accumulation of NOXA protein was significantly reduced in the Jeko1-BR clone when compared with the parental cell line Jeko1-BS whereas NOXA stabilization by Orlistat and MLN4924 was not altered in Jeko1-BR (Figure 5c, right panel).

\section{Discussion}

In this study, we provide evidence for a critical role of NOXA in the decision between life and death in MCL cells. We found a stunning discrepancy between constitutive NOXA mRNA and NOXA protein expression in MCL cell lines and primary cells. MCL cells constitutively express high levels of NOXA transcript mediated by an active PI3K/AKT/mTOR signaling pathway. In contrast, NOXA protein expression was found to be low because of rapid UPS-mediated degradation. Targeting this high NOXA protein turnover with different inhibitors of the UPS led to accumulation of NOXA protein and induction of NOXA-dependent apoptosis. Of major importance, the high constitutive NOXA mRNA expression is essential for an efficient response of MCL cells to UPS inhibitors.

NOXA is a central mediator of stress responses and critical for setting the apoptotic threshold. NOXA was initially defined as a p53-inducible gene, which is transcriptionally activated in response to cellular stress. ${ }^{16}$ More recently, it has been shown that NOXA can also be induced independently of p53 by other transcription factors such as p73 and E2F1, ${ }^{27,28}$ and has an important role in normal lymphocyte homeostasis. ${ }^{20}$ 

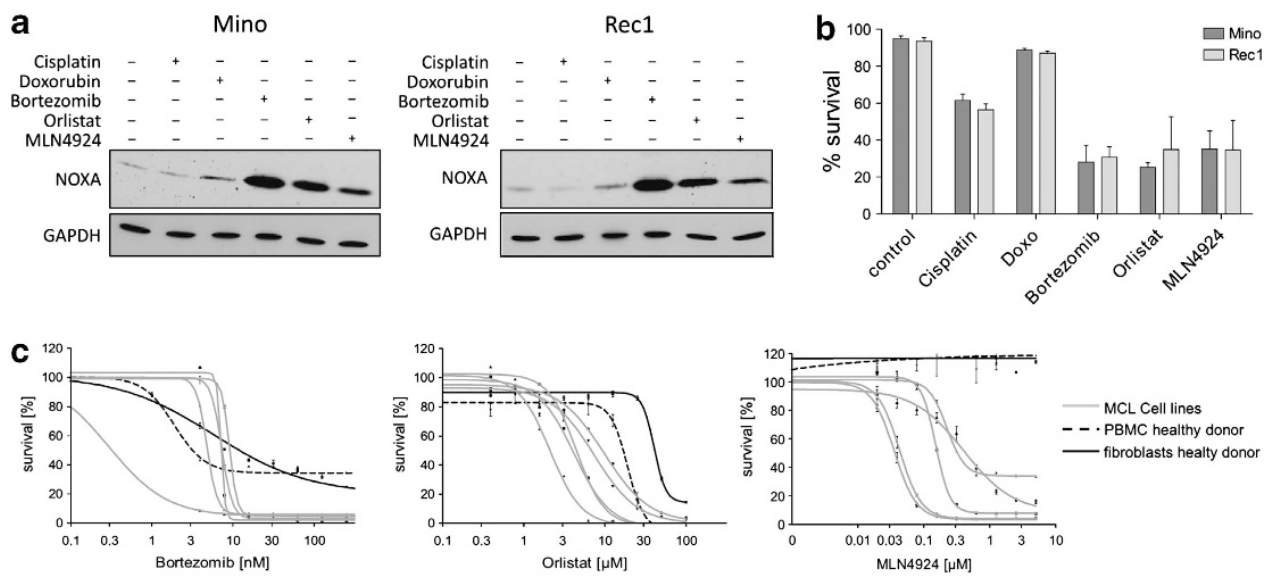

d
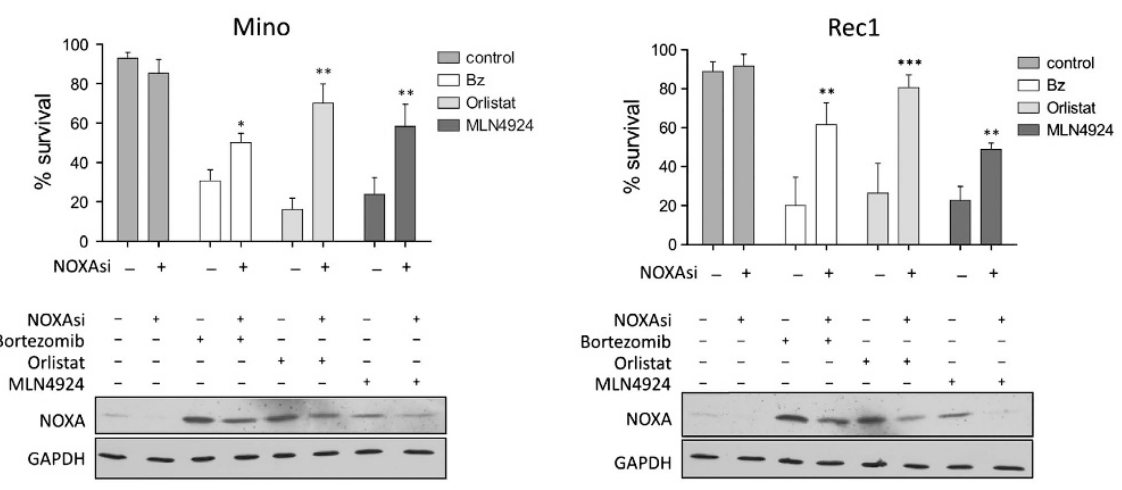

e
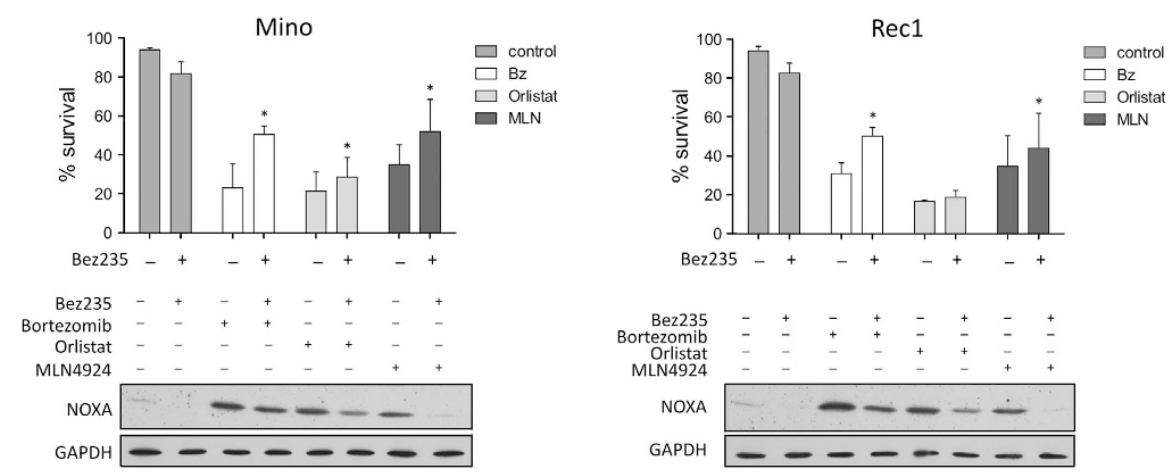

Figure 4 Agents accumulating NOXA protein efficiently induce NOXA-dependent apoptosis in MCL cells. (a) Screening for substances able to induce NOXA protein expression. MCL cell lines Mino and Rec1 were treated with Cisplatin (10 $\mu \mathrm{M})$, Doxorubicin (100 nM), Bortezomib (10 nM), Orlistat (15 $\mu \mathrm{M})$ or MLN4924 (0.5 $\mu \mathrm{M})$ for $16 \mathrm{~h}$ and NOXA protein expression was analyzed by western blot. (b) Accumulation of NOXA efficiently induces cell death. MCL cells were treated with Cisplatin (10 $\mu \mathrm{M})$, Doxorubicin $(100 \mathrm{nM})$, Bortezomib $(10 \mathrm{nM})$, Orlistat $(15 \mu \mathrm{M})$ or MLN4924 $(0.5 \mu \mathrm{M})$ for $24 \mathrm{~h}$ and cell viability was analyzed by Annexin V staining and flow cytometry. (c) Dose-response curves of MCL cell lines Mino, Jek01, Rec1, Jvm2 and Granta519 compared with PBMCs and fibroblasts of healthy donors. Cells were treated with increasing concentrations of Bortezomib, Orlistat or MLN4924 and cell viability measured by MTT. (d) NOXA siRNA rescues from Bortezomib, Orlistat and MLN4924 induced apoptosis. Cells were transfected with control siRNA or siRNA targeting NOXA. At $24 \mathrm{~h}$ after transfection, cells were treated with Bortezomib $(10 \mathrm{nM})$, Orlistat $(15 \mu \mathrm{M})$ or MLN4924 $(0.5 \mu \mathrm{M})$ and further cultivated for $24 \mathrm{~h}$. Cells were then harvested for western blot analysis and quantification of cell death by Annexin $V$ staining and flow cytometry $\left({ }^{* *} P<0.01\right.$, ${ }^{* * *} P<0.001$ ). Data represent means \pm S.D. from three experiments. (e) Active PI3K/AKT/mTOR signaling is needed for effective accumulation of NOXA protein and induction of cell death upon treatment with Bortezomib, Orlistat or MLN4924. MCL cell lines Mino and Rec1 were pre-treated with $1 \mu \mathrm{M}$ Bez235 $(6 \mathrm{~h})$ before treatment with Bortezomib $(10 \mathrm{nM})$, Orlistat $(15 \mu \mathrm{M})$ or MLN4924 $(0.5 \mu \mathrm{M})$. After $16 \mathrm{~h}$, cells were harvested for western blot analysis (lower panel). Quantification of cell death by Annexin V staining and flow cytometry was determined $24 \mathrm{~h}$ upon treatment $\left({ }^{*} P<0.05\right)$. Data represent means \pm S.D. from three experiments

It is transcriptionally upregulated upon antigen stimulation thereby regulating apoptosis of activated $\mathrm{B}$ cells and the formation of high affinity clones, ${ }^{20}$ which indicates that $\mathrm{BCR}$ signaling is important for NOXA regulation. Indeed, we here demonstrate that the constitutively high NOXA transcript levels in MCL cells depend on BCR signaling, which is chronically activated in several lymphomas including MCL. ${ }^{5}$ Furthermore, our data show for the first time that the $\mathrm{PI} 3 \mathrm{~K} / \mathrm{AKT} / \mathrm{mTOR}$ pathway is the major mediator of NOXA regulation downstream of the BCR. A role of the $\mathrm{PISK} / \mathrm{AKT} / \mathrm{mTOR}$ pathway for the regulation of NOXA was previously proposed by Mei et al. ${ }^{29}$ They demonstrated that 

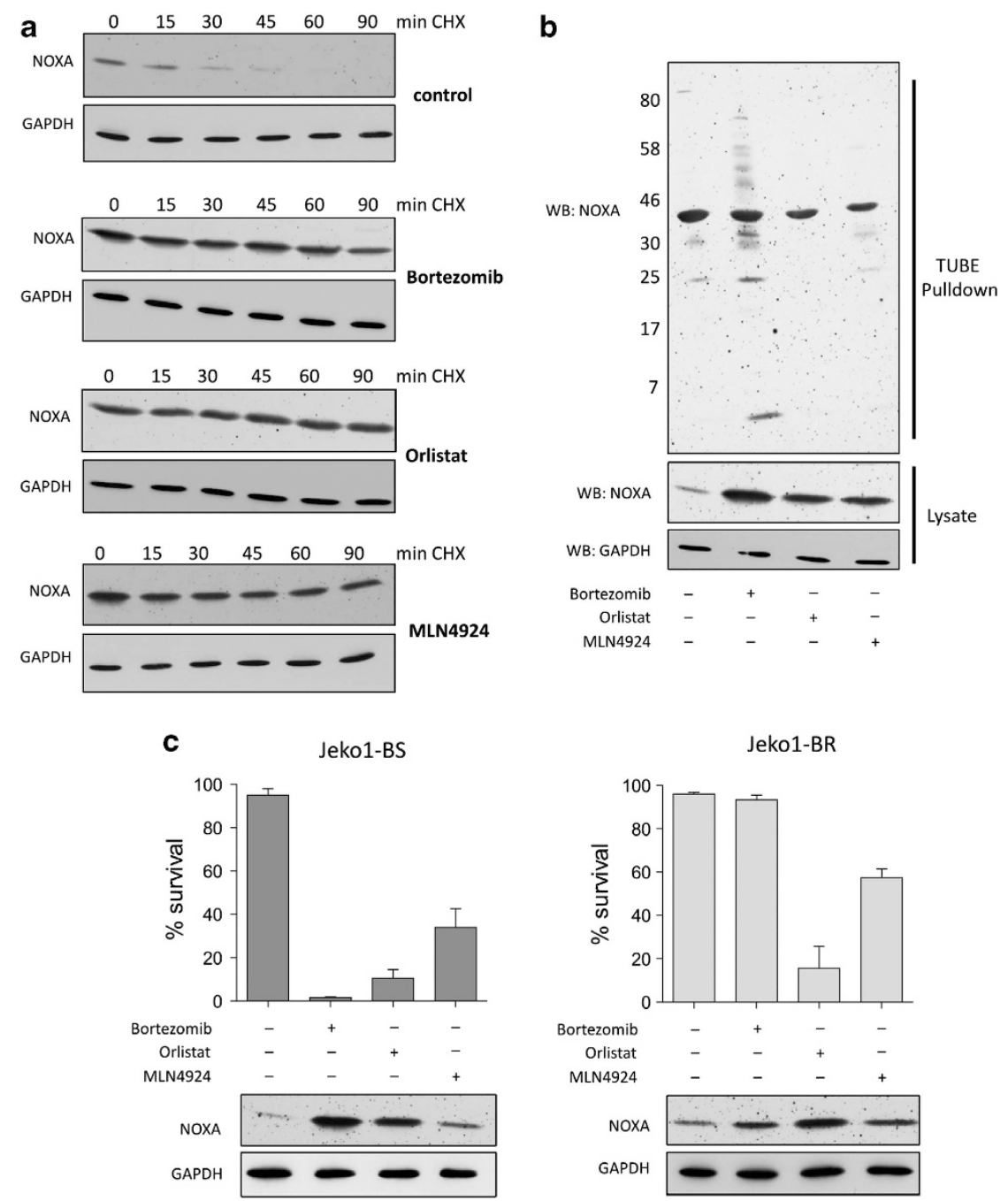

Figure 5 Orlistat and MLN4924 kill Bortezomib-resistant MCL cells by stabilizing NOXA protein in a proteasome-independent manner. (a) Bortezomib, Orlistat and MLN4924 are stabilizing NOXA protein by inhibiting rapid turnover. MCL cell line Mino was treated with Bortezomib (10 nM), Orlistat (15 $\mu \mathrm{M})$ or MLN4924 ( $0.5 \mu \mathrm{M})$ for $8 \mathrm{~h}$ and then exposed to $20 \mu \mathrm{g} / \mathrm{ml}$ cycloheximide and NOXA half-life determined by western blot. (b) Orlistat and MLN4924 stabilize NOXA by inhibiting ubiquitination of NOXA. Cells were cultivated in absence or presence of Bortezomib $(10 \mathrm{nM})$, Orlistat $(15 \mu \mathrm{M})$ or MLN4924 $(0.5 \mu \mathrm{M})$ for $8 \mathrm{~h}$ then harvest and lysed. Total polyubiquitinated proteins were isolated from cell lysates using agarose-TUBE2 beads. Protein expression and polyubiquitination state of NOXA were analyzed by western blot analysis using an NOXA-specific antibody. (c) Treatment with Orlistat and MLN4924 can kill Bortezomib-resistant MCL cells. Bortetomib-sensitive (Jeko1-BS, left panel) and Bortezomib-resistant (Jeko1-BR) MCL cells were treated for $24 \mathrm{~h}$ with Bortezomib $(10 \mathrm{nM})$, Orlistat $(15 \mu \mathrm{M})$ or MLN4924 $(0.5 \mu \mathrm{M})$ and cell viability measured by Annexin V staining and flow cytometry (upper panel). Data represent means \pm S.D. from three experiments. NOXA accumulation was determined after $8 \mathrm{~h}$ of treatment by western blot analysis (lower panel)

camptothecin-induced apoptosis in HeLa cells depends on $\mathrm{PISK} / \mathrm{AKT} / \mathrm{mTOR}$-mediated transcriptional induction of NOXA. The cAMP response element binding protein (CREB) was identified as the transcription factor mediating NOXA induction. Whether CREB has a role in MCL cells has to be investigated in further experiments. Interestingly, cyclin D1 overexpression, the hallmark of MCL, seems to contribute to the high NOXA transcript levels most likely by exerting a positive feedback loop on the PI3K/AKT/mTOR pathway.

Intriguingly, the high NOXA mRNA levels in MCL do not impair cellular survival. We found that MCL cells adapt to this constitutive pro-apoptotic signal by extensive ubiquitination and proteasomal degradation of NOXA protein. Inhibition of the proteasome using Bortezomib accumulated NOXA protein and rapidly induced apoptosis. Interestingly, active PI3K/AKT/mTOR signaling before Bortezomib treatment was needed for effective accumulation of NOXA and induction of cell death indicating that constitutively high NOXA mRNA levels are essential for the sensitivity of MCL cells to Bortezomib. Previous studies have shown that NOXA is transcriptionally activated in response to Bortezomib treatment. ${ }^{13}$ We observed only minor induction of NOXA mRNA upon Bortezomib in the MCL cell lines used in this study (data not shown) indicating that NOXA protein stabilization is the predominant mechanism of Bortezomib-mediated NOXA accumulation in MCL. Transcriptional induction of NOXA mRNA may represent a cell line dependent or secondary event in response to Bortezomib further amplifying NOXA levels.

The finding that the combination of high constitutive NOXA mRNA levels and short-lived NOXA protein is crucial for 
Bortezomib sensitivity of MCL has implications for the design of new treatment strategies. According to our results, BCR and/or PI3K/AKT/mTOR inhibitors should not be administered before Bortezomib, as active PI3K/AKT/mTOR signaling is needed for high NOXA mRNA levels and therefore constitutes a prerequisite for sensitivity to UPS inhibitors. Nevertheless, simultaneous treatment or starting with Bortezomib administration could be beneficial according to studies from other groups. ${ }^{30}$ Furthermore, intermittent treatment with Bortezomib could prevent resistance to BCR and PI3K/AKT/mTOR inhibitors by eliminating clones with active PI3K/AKT/mTOR signaling. In addition, high constitutive NOXA transcript levels may represent a predictive marker for Bortezomib sensitivity in general. The data from CCLE and other studies implicate that this phenotype may also be present in other lymphomas such as CLL and ALCL and could explain their sensitivity to Bortezomib. ${ }^{17,31}$ Chronic active BCR signaling has also been observed in CLL and could be responsible for high NOXA expression in this tumor. ${ }^{5}$ Furthermore, NOXA has also been shown to be ubiquitinated and rapidly degraded in CLL and NOXA stabilization rather than transcriptional induction has been implicated in Bortezomib-induced apoptosis in this type of Iymphoma. ${ }^{17}$ Yet, nothing is known about chronic active T-cell receptor signaling in ALCL but constitutively active $\mathrm{PI} 3 \mathrm{~K} / \mathrm{AKT} / \mathrm{mTOR}$ signaling has been described in this type of lymphoma. $^{32}$ Interestingly, the correlation between NOXA transcript levels and Bortezomib sensitivity does not seem to be limited to hematopoietic and lymphoid malignancies. Thyroid cancer cell lines expressing the highest levels of NOXA mRNA across all entities have also been shown to be sensitive to Bortezomib and the first clinical studies were promising. ${ }^{33,34}$

In addition to Bortezomib, we identified two other agents, MLN4924 and Orlistat, which stabilize and accumulate NOXA protein. Remarkably, in contrast to Bortezomib, both substances interfere with NOXA ubiquitination rather than with the proteasome as accumulation of polyubiquitinated forms of NOXA was lacking upon treatment with these inhibitors. MLN4924 is an inhibitor of neddylation, an essential process for the activity of cullin-RING ubiquitin ligases (CRLs). ${ }^{35} \mathrm{CRLS}$ are multi-subunit complexes regulating turnover of many proteins involved in cell growth and survival and members of this family have been described to be potential oncogenes. ${ }^{36}$ The hypothesis that NOXA might be regulated by this family of ubiquitin ligases is supported by the finding that overexpression of SAG, a subunit of the SCF E3 ligase subfamily of CRLs, reduces NOXA stability and confers radiation resistance in lung carcinoma and glioblastoma. ${ }^{37}$ Orlistat, the second substance found to stabilize NOXA in our study, is an inhibitor of the FASN, an essential enzyme in palmitate synthesis and lipogenesis. FASN is overexpressed in many malignancies including MCL. ${ }^{24,38}$ Interestingly, Orlistat was shown to be effective in MCL cell lines and primary MCL cells. ${ }^{24}$ Here, we demonstrate that Orlistat-mediated FASN inhibition induces apoptosis by impairing NOXA ubiquitination. It has been shown that FASN inhibition reduces the expression of components of a subgroup of $\mathrm{CRLs}^{39}$ and may also indirectly target the ubiquitin ligase of NOXA. Together, our data obtained with MLN4924 and Orlistat suggest that increased constitutive polyubiquitination and degradation of NOXA is mediated by enhanced activity of a certain CRL E3 ligase complex in MCL. Further experiments are warranted to identify the ubiquitin ligase of NOXA and/or the CRL component involved in NOXA turnover. A deeper insight into the mechanism and regulation of NOXA ubiquitination may lead to the identification of novel targets for more efficient and selective treatment not only of MCL. This view is supported by our data demonstrating that both MLN4924 and Orlistat seem to block growth more selectively in MCL than Bortezomib. Although Orlistat appears to be selective for cyclin D1-overexpressing cells, our data indicate that MLN4924 has a more general selectivity to tumor cells. MLN4924 may be indeed a promising anticancer agent not only for treatment of MCL patients. Targeting CRL activity has already shown promising effects in different types of tumors including lymphomas. ${ }^{40,41}$

Furthermore, Bortezomib-resistant MCL cells were still sensitive to either Orlistat or MLN4924. Both inhibitors were able to induce NOXA in Bortezomib-resistant clones probably through targeting UPS-mediated NOXA turnover upstream of the proteasome.

Interestingly, NOXA stabilization upon MLN4924 was not as strong as observed after Bortezomib or Orlistat although efficacy of NOXA-dependent cell death of the three substances was comparable. Cellular levels of Mcl1 can influence the susceptibility of cells to NOXA-induced apoptosis. ${ }^{16} \mathrm{We}$ here demonstrate that in contrast to Bortezomib or Orlistat, which accumulate Mcl1 in parallel to NOXA, MLN4924 treatment of the lymphoma cells rather reduces the levels of the anti-apoptotic protein. This observation indicates that less NOXA protein accumulation is sufficient to efficiently induce cell death upon treatment with MLN4924.

In conclusion, our data demonstrate that the discrepancy between NOXA mRNA and NOXA protein levels may represent an important Achilles' heel of $M C L$, and offers novel options for the improvement of $\mathrm{MCL}$ treatment. We found that the PI3K/AKT/mTOR pathway mediated high constitutive NOXA mRNA levels and stabilization of the short-lived NOXA protein are critical determinants of Bortezomib sensitivity in MCL. Furthermore, we identified alternative substances stabilizing NOXA independently of proteasome inhibition thereby offering novel treatment strategies to Bortezomib-resistant cells.

\section{Materials and Methods}

Cell lines and patient samples. The MCL cell lines Mino, Jeko1, Rec1, Jvm2 and Granta519 were kind gifts from A Rosenwald, Department of Pathology, University of Würzburg, Germany. A Bortezomib-resistant (BR) subclone of Jeko1 was generated by continuous cultivation of the parental cell line in increasing Bortezomib concentrations. 2102EP was kindly provided by T Mueller, Department of Oncology, University of Halle, Germany. NCl-H460, A549 and NCl-H23 cells were procured from ATCC (Manassas, VA, USA). OVCAR5, SKOV3 and A2780 were obtained from the NCl-60 cell panel. NTERA2/D1 cells were purchased from LGC Standards, Wesel, Germany. All cells were cultivated in RPMI- 1640 (Biochrom, Berlin, Germany) with 10\% FCS and glutamine. The local ethics committee approved the collection of patient and healthy donor samples (project number 159/2011BO2) and informed consent was obtained from the patients in accordance with the Declaration of Helsinki. Primary MCL cells were obtained from lymph nodes biopsies of four patients. Extracted cells were cultivated in X-VIVO 10 medium (Lonza, Cologne, Germany) supplemented with 10\% human serum.

Antibodies and reagents. (Ser473)-Akt, (Ser235/236)-S6, (Ser32/36)-IкB, (Thr202/Tyr204)-Erk1/2, Akt, S6, Erk1/2, IkB, p65, CD79A, Mcl1 and GAPDH 
antibodies were purchased from Cell Signaling, Danvers, MA, USA. Anti-NOXA was obtained from Calbiochem, La Jolla, CA, USA. Bez235, Ibrutinib, PD0325901, MG132, Bortezomib, LY294002 and BKM120 were obtained from Selleckchem, Houston, TX, USA. Temsirolimus, Orlistat and cycloheximide were purchased from Sigma, Munich, Germany. MLN4924 was obtained from ChemieTek, Indianapolis, IN, USA.

Analysis of protein expression. Cells were lysed according to standard protocols. Western blot was performed as described previously. ${ }^{42}$

Analysis of mRNA expression. Total RNA was extracted and transcribed to cDNA according to standard protocols. NOXA expression was analyzed using TaqMan Gene Expression Assays Hs00560402_m1 (NOXA/PMAIP1), Hs02758991_g1 (GAPDH) and Hs00427620_m1 (TBP) (Applied Biosystems, Norwalk, CT, USA) on BioMark HD System (Fluidigm, South San Francisco, CA USA) or 7900 HT Fast Real-Time PCR System (Applied Biosystems) according to the manufacturer's instructions. TBP was used for normalization of NOXA mRNA expression analyzed by high-throughput BioMark HD System, which includes a pre-amplification step. GAPDH was used for normalization of NOXA mRNA expression analyzed by conventional real-time PCR using 7900 HT Fast Real-Time PCR System.

Detection of cell death. Induction of cell death was assessed by Annexin V (BD Pharmingen, Heidelberg, Germany) staining according to the manufacturer's instructions and analyzed by flow cytometry.

Growth inhibition analysis. Growth inhibition was assessed from changes in mitochondrial activity after $48 \mathrm{~h}$ upon treatment using MTT assay. ${ }^{43}$

siRNA experiments. For silencing, we used siGenome SMARTpool small interfering ribonucleic acid (siRNA) (Thermo Fisher Scientific, Dharmacon, Lafayette, CO, USA). Sequences targeted by SMARTpool siRNAs are described in Supplementary Table 1. As a control, we used Non-Targeting siRNA\#1. Cells were transfected by electroporation using a single-pulse protocol $(250 \mathrm{~V}, 1800 \mu \mathrm{F}$, $\infty \mathrm{W})$. At $16-48 \mathrm{~h}$ upon electroporation, cells were treated according to requirements. To evaluate knockdown efficacy, protein lysates were obtained and analyzed by western Blot.

Pull-down of polyubiquitinated proteins. Cells were lysed by standard protocols and pull-down of polyubiquitinated proteins was performed using agarose-TUBE2 (Tebu-Bio, Offenbach, Germany) according to the manufacturer's instructions and analyzed by western Blot.

Analysis of NOXA protein half-life. The half-life of NOXA was determined by exposing the cells to $20 \mu \mathrm{g} / \mathrm{ml}$ cycloheximide inhibiting protein synthesis. For western blot analysis, cells were harvested and lysed after indicated times.

\section{Conflict of Interest}

The authors declare no conflict of interest.

Acknowledgements. We thank Andreas Rosenwald (Department of Pathology, Würzburg, Germany) and Thomas Mueller (Department of Oncology, Halle, Germany) for kindly providing the MCL cell lines and the embryonal carcinoma cell line 2102EP. We are grateful to Kerstin Willecke for excellent technical assistance. This work was financially supported by the Robert Bosch Foundation (project $\mathrm{O} 2$ and $\mathrm{O3}$ ).

1. Jares P, Colomer D, Campo E. Genetic and molecular pathogenesis of mantle cell lymphoma: perspectives for new targeted therapeutics. Nat Rev Cancer 2007; 7: 750-762.

2. Jares $P$, Colomer $D$, Campo $E$. Molecular pathogenesis of mantle cell lymphoma. J Clin Invest 2012; 122: 3416-3423

3. Pérez-Galán $P$, Dreyling $M$, Wiestner $A$. Mantle cell lymphoma: biology, pathogenesis, and the molecular basis of treatment in the genomic era. Blood 2011; 117: 26-38.

4. Fernàndez V, Hartmann $E$, Ott G, Campo $E$, Rosenwald A. Pathogenesis of mantle-cell lymphoma: all oncogenic roads lead to dysregulation of cell cycle and DNA damage response pathways. J Clin Oncol 2005; 23: 6364-6369.

5. Young RM, Staudt LM. Targeting pathological B cell receptor signalling in lymphoid malignancies. Nat Rev Drug Discov 2013; 12: 229-243.
6. Col JD, Zancai P, Terrin L, Guidoboni M, Ponzoni M, Pavan A et al. Distinct functional significance of Akt and mTOR constitutive activation in mantle cell lymphoma. Blood 2008; 111: 5142-5151.

7. Martínez N, Camacho Fl, Algara P, Rodríguez A, Dopazo A, Ruíz-Ballesteros E et al. The molecular signature of mantle cell lymphoma reveals multiple signals favoring cell survival. Cancer Res 2003; 63: 8226-8232.

8. Rummel MJ, de Vos S, Hoelzer D, Koeffler HP, Hofmann W-K. Altered apoptosis pathways in mantle cell lymphoma. Leuk Lymphoma 2004; 45: 49-54.

9. Tagawa H, Karnan S, Suzuki R, Matsuo K, Zhang X, Ota A et al. Genome-wide array-based CGH for mantle cell lymphoma: identification of homozygous deletions of the proapoptotic gene BIM. Oncogene 2005; 24: 1348-1358.

10. Wang ML, Rule S, Martin P, Goy A, Auer R, Kahl BS et al. Targeting BTK with Ibrutinib in relapsed or refractory mantle-cell lymphoma. N Engl J Med 2013; 369: 507-516.

11. Kim A, Park S, Lee J-E, Jang W-S, Lee S-J, Kang HJ et al. The dual PI3K and mTOR inhibitor NVP-BEZ235 exhibits anti-proliferative activity and overcomes bortezomib resistance in mantle cell lymphoma cells. Leuk Res 2012; 36: 912-920.

12. Chen D, Frezza M, Schmitt S, Kanwar J, Dou QP. Bortezomib as the first proteasome inhibitor anticancer drug: current status and future perspectives. Curr Cancer Drug Targets 2011; 11: 239-253.

13. Pérez-Galán P, Roué G, Villamor N, Montserrat E, Campo E, Colomer D. The proteasome inhibitor bortezomib induces apoptosis in mantle-cell lymphoma through generation of ROS and Noxa activation independent of p53 status. Blood 2006; 107: 257-264.

14. Youle RJ, Strasser A. The BCL-2 protein family: opposing activities that mediate cell death. Nat Rev Mol Cell Biol 2008; 9: 47-59.

15. Adams J, Cory S. The Bcl-2 apoptotic switch in cancer development and therapy. Oncogene 2007; 26: 1324-1337.

16. Ploner C, Kofler R, Villunger A. Noxa: at the tip of the balance between life and death. Oncogene 2008; 27(Suppl 1): S84-S92.

17. Baou M, Kohlhaas SL, Butterworth M, Vogler M, Dinsdale D, Walewska R et al. Role of NOXA and its ubiquitination in proteasome inhibitor-induced apoptosis in chronic lymphocytic leukemia cells. Haematologica 2010; 95: 1510-1518.

18. Brinkmann K, Zigrino P, Witt A, Schell M, Ackermann L, Broxtermann P et al. Ubiquitin C-terminal hydrolase-L1 potentiates cancer chemosensitivity by stabilizing NOXA. Cell Rep 2013; 3: 881-891.

19. Gutekunst M, Mueller T, Weilbacher A, Dengler MA, Bedke J, Kruck S et al. Cisplatin hypersensitivity of testicular germ cell tumors is determined by high constitutive Noxa levels mediated by Oct-4. Cancer Res 2013; 73: 1460-1469.

20. Wensveen FM, Derks IAM, van Gisbergen KPJM, de Bruin AM, Meijers JCM, Yigittop H et al. $\mathrm{BH} 3-$ only protein Noxa regulates apoptosis in activated $\mathrm{B}$ cells and controls high-affinity antibody formation. Blood 2012; 119: 1440-1449.

21. Bretz J, Garcia J, Huang X, Kang L, Zhang Y, Toellner K-M et al. Noxa mediates p18INK4C cell-cycle control of homeostasis in B cells and plasma cell precursors. Blood 2011; 117: 2179-2188.

22. Barretina J, Caponigro G, Stransky N, Venkatesan K, Margolin AA, Kim S et al. The Cancer Cell Line Encyclopedia enables predictive modelling of anticancer drug sensitivity. Nature 2012; 483: 603-607.

23. Holkova B, Grant S. Proteasome inhibitors in mantle cell lymphoma. Best Pract Res Clin Haematol 2012; 25: 133-141

24. Gelebart $\mathrm{P}$, Zak Z, Anand $\mathrm{M}$, Belch $\mathrm{A}$, Lai R. Blockade of fatty acid synthase triggers significant apoptosis in mantle cell lymphoma. PloS One 2012; 7: e33738.

25. Gutekunst M, Oren M, Weilbacher A, Dengler MA, Markwardt C, Thomale J et al. p53 hypersensitivity is the predominant mechanism of the unique responsiveness of testicular germ cell tumor (TGCT) cells to cisplatin. PloS One 2011; 6: e19198.

26. Kurata K, Yanagisawa R, Ohira M, Kitagawa M, Nakagawara A, Kamijo T. Stress via p53 pathway causes apoptosis by mitochondrial Noxa upregulation in doxorubicin-treated neuroblastoma cells. Oncogene 2008; 27: 741-754.

27. Flinterman M, Guelen L, Ezzati-Nik S, Killick R, Melino G, Tominaga K et al. E1A activates transcription of p73 and Noxa to induce apoptosis. J Biol Chem 2005; 280: 5945-5959.

28. Hershko T, Ginsberg D. Up-regulation of Bcl-2 homology $3(\mathrm{BH} 3)$-only proteins by E2F1 mediates apoptosis. J Biol Chem 2004; 279: 8627-8634.

29. Mei Y, Xie C, Xie W, Tian X, Li M, Wu M. Noxa/Mcl-1 balance regulates susceptibility of cells to camptothecin-induced apoptosis. Neoplasia 2007; 9: 871-881.

30. Dasmahapatra G, Patel H, Dent P, Fisher RI, Friedberg J, Grant S. The Bruton tyrosine kinase (BTK) inhibitor $\mathrm{PCl}-32765$ synergistically increases proteasome inhibitor activity in diffuse large-B cell lymphoma (DLBCL) and mantle cell lymphoma (MCL) cells sensitive or resistant to bortezomib. Br J Haematol 2013; 161: 43-56.

31. Bonvini $\mathrm{P}$, Zorzi E, Basso G, Rosolen A. Bortezomib-mediated $26 \mathrm{~S}$ proteasome inhibition causes cell-cycle arrest and induces apoptosis in CD-30 + anaplastic large cell lymphoma. Leukemia 2007; 21: 838-842.

32. Vega F, Medeiros LJ, Leventaki V, Atwell C, Cho-Vega JH, Tian L et al. Activation of mammalian target of rapamycin signaling pathway contributes to tumor cell survival in anaplastic lymphoma kinase-positive anaplastic large cell lymphoma. Cancer Res 2006; 66: 6589-6597.

33. Mitsiades CS, McMillin D, Kotoula V, Poulaki V, McMullan C, Negri J et al. Antitumor effects of the proteasome inhibitor bortezomib in medullary and anaplastic thyroid carcinoma cells in vitro. J Clin Endocrinol Metab 2006; 91: 4013-4021. 
34. Putzer D, Gabriel M, Kroiss A, Madleitner R, Eisterer W, Kendler D et al. First experience with proteasome inhibitor treatment of radioiodine nonavid thyroid cancer using bortezomib. Clin Nucl Med 2012; 37: 539-544.

35. Soucy TA, Smith PG, Milhollen MA, Berger AJ, Gavin JM, Adhikari S et al. An inhibitor of NEDD8-activating enzyme as a new approach to treat cancer. Nature 2009; 458: 732-736.

36. Wei S, Chu P-C, Chuang H-C, Hung W-C, Kulp SK, Chen C-S. Targeting the oncogenic E3 ligase Skp2 in prostate and breast cancer cells with a novel energy restriction-mimetic agent. PLoS One 2012; 7: e47298.

37. Jia L, Yang J, Hao X, Zheng M, He H, Xiong X et al. Validation of SAG/RBX2/ROC2 E3 ubiquitin ligase as an anticancer and radiosensitizing target. Clin Cancer Res 2010; 16: 814-824.

38. Flavin R, Peluso S, Nguyen PL, Loda M. Fatty acid synthase as a potential therapeutic target in cancer. Future Oncol Lond Engl 2010; 6: 551-562.

39. Knowles LM, Axelrod F, Browne CD, Smith JW. A fatty acid synthase blockade induces tumor cell-cycle arrest by down-regulating Skp2. J Biol Chem 2004; 279: 30540-30545.

40. Milhollen MA, Traore T, Adams-Duffy J, Thomas MP, Berger AJ, Dang L et al. MLN4924, a NEDD8-activating enzyme inhibitor, is active in diffuse large B-cell lymphoma models: rationale for treatment of NF-KB-dependent lymphoma. Blood 2010; 116 1515-1523.

41. Swords RT, Kelly KR, Smith PG, Garnsey JJ, Mahalingam D, Medina E et al. Inhibition of NEDD8-activating enzyme: a novel approach for the treatment of acute myeloid leukemia. Blood 2010; 115: 3796-3800.

42. Dengler MA, Staiger AM, Gutekunst M, Hofmann U, Doszczak M, Scheurich $P$ et al Oncogenic stress induced by acute hyper-activation of Bcr-Abl leads to cell death upon induction of excessive aerobic glycolysis. PLOS One 2011; 6: e25139.

43. Kuip H, van der, Goetz AW, Miething C, Duyster J, Aulitzky WE. Adhesion to fibronectin selectively protects Bcr-Abl + cells from DNA damage-induced apoptosis. Blood 2001; 98: $1532-1541$.

(c) (i) $\Theta$ Cell Death and Disease is an open-access journal published by Nature Publishing Group. This work is licensed under a Creative Commons Attribution-NonCommercialNoDerivs 3.0 Unported License. To view a copy of this license, visit http://creativecommons.org/licenses/by-nc-nd/3.0/

Supplementary Information accompanies this paper on Cell Death and Disease website (http://www.nature.com/cddis) 